\title{
An improved prism energy analyzer for neutrons
}

\author{
J. Schulz ${ }^{1 \mathrm{a}}$, F. Ott ${ }^{\mathrm{b}}$, Th. Krist ${ }^{\mathrm{a}}$ \\ ${ }^{a}$ Helmholtz-Zentrum Berlin für Materialien und Energie, Hahn-Meitner-Platz 1, 14109 \\ Berlin, Germany \\ ${ }^{b}$ Laboratoire Leon Brillouin, Bât 563 CEA Saclay, 91191 Gif sur Yvette Cedex, France
}

\begin{abstract}
The effects of two improvements of an existing neutron energy analyzer consisting of stacked silicon prism rows are presented. Firstly we tested the effect of coating the back of the prism rows with an absorbing layer to suppress neutron scattering by total reflection and by refraction at small angles. Experiments at HZB showed that this works perfectly. Secondly the prism rows were bent to shift the transmitted wavelength band to larger wavelengths. At HZB we showed that bending increased the transmission of neutrons with a wavelength of $4.9 \AA$. Experiments with a white beam at the EROS reflectometer at LLB showed that bending of the energy analyzing device allows to shift the transmitted wavelength band from 0 to $9 \AA$ to 2 to $16 \AA$.
\end{abstract}

Keywords: Neutron Optics, Energy analysis, Prisms

\section{Introduction}

The proposed neutron energy analyzer is used to measure at different wavelengths at the same time and so to enhance the usable flux e.g. in a reflectometer used in time-of-flight mode. In the last years several energy analyzing devices had been developed, tested and improved (summarized in [1],[2]) e.g. refraction based devices such as very flat prisms [3] and stacked prism systems [5]. Flat prisms take advantage of the refraction at small angles which are several orders of magnitude higher than the refraction at angles around $45 \mathrm{deg}$ [4]. For neutrons that are refracted at 0.2 deg the deflection is in the range of $10^{-1} \mathrm{deg}$ whereas the refraction by a single prism

\footnotetext{
${ }^{1}$ Corresponding author. Tel.: +49 30806243048.

E-mail address: jennifer.schulz@helmholtz-berlin.de (J. Schulz)
} 
with side walls at an angle of $54.7 \mathrm{deg}$ is only $6 \cdot 10^{-4} \mathrm{deg}$ for $4.9 \AA$ neutrons. Thus a large number of such prisms is neccessary to achieve a good wavelength resolution.

The advantage of such a prism system is its flexibility. More prisms can be added to achieve higher deflections and to encode broader beams.

The disadvantages of a system with stacked prism layers are the total reflection and the refraction at small angles which occur when neutrons interact with the back of the prisms in the next prism layer at an angle close to the critical angle of the total reflection. Since in both cases the neutrons are scattered in opposite direction compared to the one of the refraction at the sides of the prisms these neutrons are lost for the energy analysis and are counted as background. They lead at the detector to an intensity increase at small wavelengths and make the use of the device in a reflectometry measurement less useful [5]. These background neutrons can be removed by coating the back of the prisms with an absorbing layer, cf. $\S 3$.

A flat prism row with 191 prisms and a height of $0.25 \mathrm{~mm}$ transmits neutrons in a wavelength band from $0 \AA$ to $9 \AA$ [5]. To increase the transmission of neutrons with larger wavelengths a bending, which follows the deflection of the neutrons inside the prism rows can be applied (see Fig.1). This way neutrons with larger wavelengths can pass through the prism rows without interacting with the back of the prisms in the next row. The intensity is maximal for neutrons with such a wavelength that their path follows the bending of the prism stack. Neutrons outside a wavelength band around this ideal wavelength get absorbed when they hit the coated back of the prisms below or above their entry row. VITESS [6] simulations show that the wavelength band can be shifted to longer wavelengths by increasing the bending angle to values between 1 and $10 \mathrm{~m}$ (see Fig.2). This way the wavelength band from 0 to $20 \AA$ can be covered with 5 different bending angles. It should be noted that the widths of the wavelength bands become smaller for increasing wavelengths since the refraction angle is proportional to the square of the neutron wavelength. The experimental verification of this idea is shown in $\S 4$.

\section{Theory}

In this section we want to derive a formula for the attenuation of a neutron beam in a bent prism energy analyzer where the backs of the prisms are 
coated with an absorbing material.

For the prism rows the refraction angle and thus the wavelength resolution increases with the number of prisms. The angular dispersion of the system is the wavelength dependence of the refraction angle according to the wavelength dependence in the index of refraction $n$, which is

$$
n=1-\frac{\lambda^{2}}{2 \pi} N b
$$

where $\lambda$ is the wavelength of the neutron and $N b$ the scattering length density of the prism material. The refraction angle $\varphi$ is given by Snells law

$$
\varphi=\alpha-\sin ^{-1}(n \sin \alpha)
$$

where $\alpha$ is the incident angle.

The absorption in the material is given by the linear attenuation coefficient $\mu$ and the material thickness:

$$
d=\frac{b}{2} \cdot i
$$

where $b$ is the base width and $i$ the number of the prisms. For the whole prism system this leads to a transmission of

$$
T_{\text {Abs. }}=e^{-\mu \cdot \frac{b}{2} \cdot i}=e^{-\mu \cdot \frac{i h}{\tan (\Phi)}},
$$

where $h$ is the constant height of the prisms and $\Phi$ is the angle between the base and the sides of the prisms.

The absorption due to losses at the back of the prisms is given by the number of neutrons which arrive at the back of the prisms in the next layer. This is given by the geometry of the prism system and the refraction per prism. For small wavelengths only neutrons close to the next prism layer will touch it. For a fixed number of prisms the deflection in height, $\Delta_{z}$, of the neutrons inside the prism rows should be calculated here. Assuming the neutrons enter the prism rows parallel to the prism bases, homogeneously distributed over the whole height and without divergence the ratio of $\Delta_{z} / h$ gives the losses due to absorption at the absorbing layer at the back of the prisms. For a single prism the deflection is given by:

$$
\Delta_{z_{1}}=b \cdot \tan \Theta
$$


with the refraction angle $\Theta=\varphi_{\text {out }}+\varphi_{\text {in }}$ for a single prism and $\varphi_{\text {in }}$ and $\varphi_{\text {out }}$ the refraction angles of the neutrons when entering and leaving the prism according to eq.2.

At each prism the angle changes as well as the deflection. The total deflection for a prism row with i prisms is given by:

$$
\begin{aligned}
\Delta_{z} & =\sum_{j=1}^{i}\left(j b \cdot \tan \left(\Theta_{j}\right)\right) \approx b \cdot \tan \left(\Theta_{1}\right) \sum_{j=1}^{i} j \\
& =\frac{i^{2}+i}{2} b \cdot \tan \left(\Theta_{1}\right) .
\end{aligned}
$$

For all practical cases this is a very good approximation since for $\alpha=54.7 \mathrm{deg}$ and 1000 prisms the relative error is $3 \cdot 10^{-4}$ for $4.9 \AA$ and $2 \cdot 10^{-2}$ for $20 \AA$. The fraction of neutrons that do not hit the back of the next prism row is:

$$
T_{\text {Refl. }}=1-\frac{\Delta_{z}}{h}
$$

For curved prism rows the expression changes to:

$$
T_{\text {Refl. }}=1-\frac{\left|\Delta_{z}-x\right|}{h},
$$

where $\mathrm{x}$ is the deflection of the prism row due to the bending. For $\left|\Delta_{z}-x\right|>h$ all neutrons are absorbed.

The whole transmission is the product of both effects:

$$
T=T_{\text {Refl. }} \cdot T_{\text {Abs. }}
$$

The function $\mathrm{T}$ is given in Fig. 2 for prism rows of 191 prisms which are bent to different curving radii.

\section{Back coating}

To check the effect of an absorbing coating the back of the prisms was coated with Gadolinium. The effect of this coating was determined at the V14 reflectometer at the HZB by measuring two energy analyzers, one with and one without an absorption layer. We used a $20 \mathrm{~mm}$ x $0.3 \mathrm{~mm}$ neutron beam with a wavelength of $4.9 \AA$. With a ${ }^{3} \mathrm{He}$ detector in $2 \mathrm{~m}$ distance the refraction by a stack of 4 prism rows each with 191 Si prisms was measured, 
see Fig. 3. For both systems the position of the maximum of the deflected intensity at $0.12 \mathrm{deg}$ relative to the incoming beam is in good agreement with the theoretical value. The small difference between the positions of the maxima of $0.005 \mathrm{deg}$ is due to small differences in the mechanical setup. For the uncoated prisms the total reflection and the refraction at small angles of the neutrons leads to an increased background at negative angles, see Fig.3. This background is eliminated by using the Gd coated prism rows. Absorbing back coating is thus a means to clear up the signal by avoiding total reflection and refraction at small angles.

\section{Bending the prism stack}

First we measured the effect of the bending at a fixed wavelength of $4.9 \AA$ at the V14 reflectometer. For this purpose an energy analyzer consisting of a stack of 4 Gd coated prism rows each with $191 \mathrm{Si}$ prisms in a simple mechanical bender had been adjusted parallel to the $20 \mathrm{~mm} \times 0.3 \mathrm{~mm}$ neutron beam. The divergence of the beam was limited to $7 \mathrm{mdeg}$. A $3 \mathrm{~mm}$ slit was put in front of the detector, which was located $2 \mathrm{~m}$ behind the energy analyzer. Thus it covers an angular range of 0.08 deg. The detector angle was set to $0.12 \mathrm{deg}$ according to the refraction by the prisms for the chosen wavelength. Then the intensity of the refracted beam was measured for different bending radii of the energy analyzer between $19.8 \mathrm{~m}$ and $42.9 \mathrm{~m}$.

Without bending the device the transmission is about $71 \%$. Bending the energy analyzer to a radius of $42.9 \mathrm{~m}$ leads to an increase of transmission (see Fig.4) to $86 \%$. The maximum transmission is $87 \%$ and was found at a bending radius of $32.4 \mathrm{~m}$, for which the total refraction angle of about $0.12 \mathrm{deg}$ equals the total bending angle of the prism rows. The intensity decreases again for radii smaller than $25.0 \mathrm{~m}$ due to the increased number of neutrons that hit the coated base sides of the prisms.

This maximum of transmission is in good agreement with the attenuation in the pure material, which is $88 \%$ for a material thickness of $3.8 \mathrm{~cm}$ and a wavelength of $4.9 \AA[7]$.

Since this agreement can only be achieved when the bending angle equals the angle of refraction this effect is strongly chromatic. Therefore another measurement with a white neutron beam was performed at the EROS reflectometer at LLB to get the wavelength depending transmission of the bent device. The bending radius of the prism stack was $7.9 \mathrm{~m}$ which corresponds to a bending angle of $0.5 \mathrm{deg}$. With such a small bending radius according 
to VITESS simulations a wavelength band between $4 \AA$ and $14 \AA$ should be transmitted. A $20 \mathrm{~mm} \times 0.3 \mathrm{~mm}$ neutron beam with a collimation of $0.01 \mathrm{deg}$ was used and the detector was set $2 \mathrm{~m}$ behind the energy analyzer.

Compared to the unbent energy analyzer where the wavelength spectrum ends at $9 \AA$ the measured spectrum of the bent device shows a shift to the intervall between $2 \AA$ and $15 \AA$. Fig. 5 shows the wavelength distribution of the neutron intensity transmitted through the bent analyzer normalized to the incoming one. The maximum of transmission is $77 \%$ for $9 \AA$ neutrons. The theoretical value for the transmission in the pure material is $81.5 \%$.

\section{Conclusion}

Coating the back of the prism rows with an absorbing layer removes the totally reflected and at small angles refracted neutrons from the spectrum of the refracted neutron beam. Additional bending of the prism rows increases the transmission for specific wavelength bands. For neutrons at the central wavelength and without divergence the remaining losses are resulting only from the attenuation in the material.

\section{References}

[1] F. Ott, A. Menelle, Eur. Phys. J. Scecial Topics 167, 93 (2009)

[2] F. Ott, A. de Vismes, Physica B 397, 153 (2007)

[3] R. Cubitt, et al., Nucl. Instrum. Meth. A 558, 547 (2006)

[4] R. Cubitt, et al., Eur. Phys. L Plus 126, 111 (2011)

[5] J. Schulz, et al., Nucl. Instrum. Meth. A accepted manuscript (2013)

[6] VITESS web site: http://www.helmholtzberlin.de/forschung/grossgeraete/neutronenstreuung/projekte/vitess/index_de.html HZB (2012)

[7] A. W. Freund, Nucl. Instrum. Methods, 213, 495-501 (1983) 


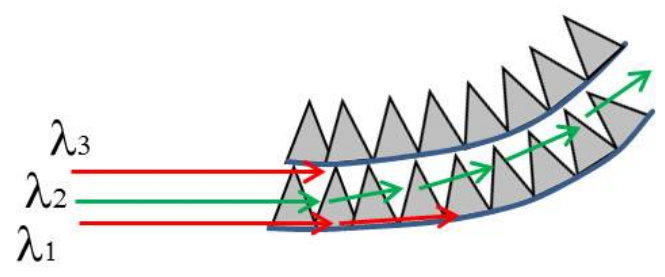

Figure 1: By bending the prism rows the transmission can be optimized for a wavelength band. Neutrons outside this wavelength band are removed by an absorbing coating at the back of the prisms.

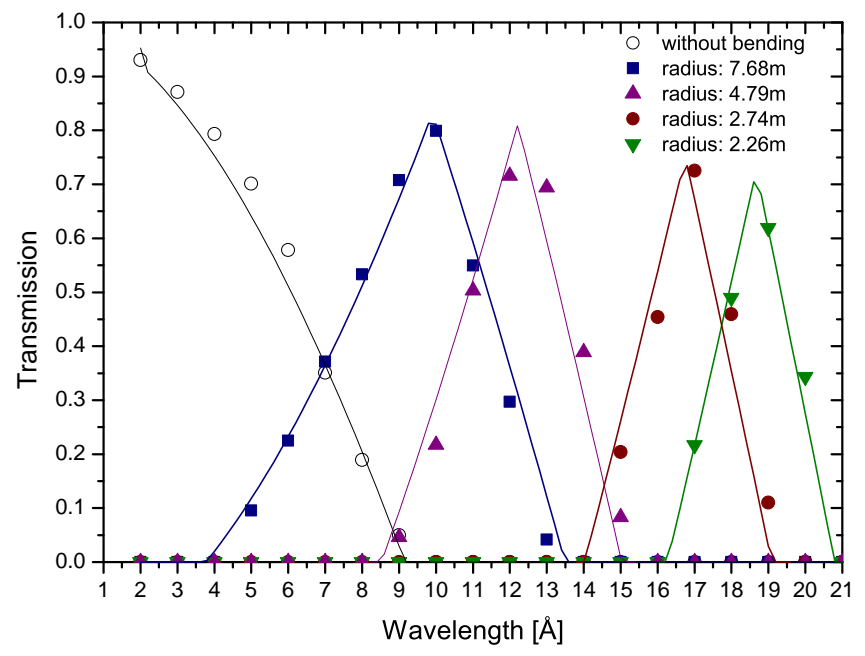

Figure 2: VITESS simulations (symbols) show that by bending prism rows with $191 \mathrm{Si}$ prisms to smaller radii the transmitted wavelength band can be shifted to higher wavelengths. The curves are calculated with eq. 10. 


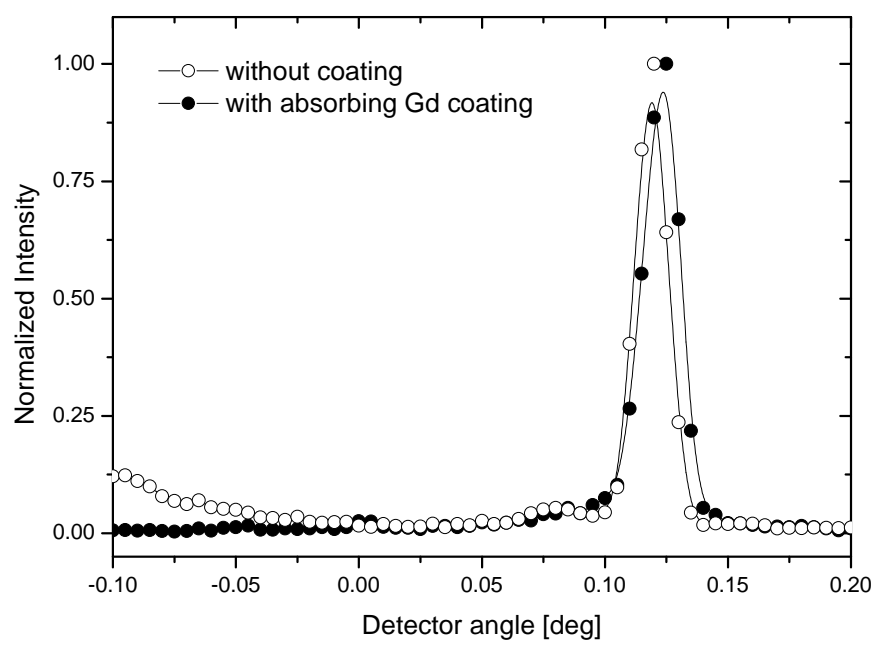

Figure 3: Measurement of the refraction of neutrons with a wavelength of $4.9 \AA$ by two energy analyzers consisting of $191 \mathrm{Si}$ prisms, one with and one without an absorbing back coating. The intensity is normalized to the same maximum height of the intensity distribution due to the different experimental settings. 


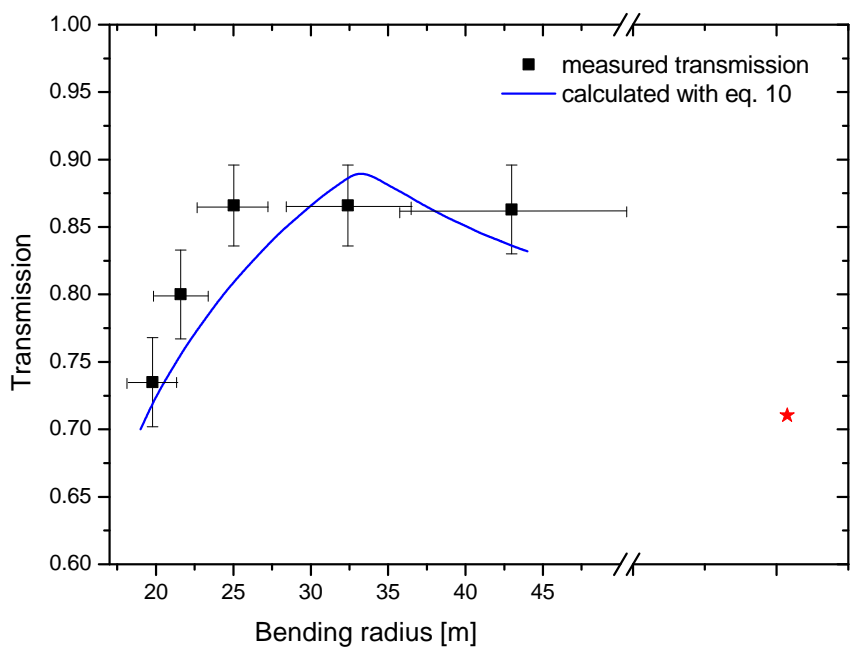

Figure 4: Measured and calculated transmission of neutrons with a wavelength of $4.9 \AA$ through an energy analyzer at different bending radii. At the maximum of transmission the bending angle of the prism rows equals the angle of refraction for $191 \mathrm{Si}$ prisms. The measured transmission for the unbent energy analyzer is shown after the break in the $\mathrm{x}$-axis as a red star. 


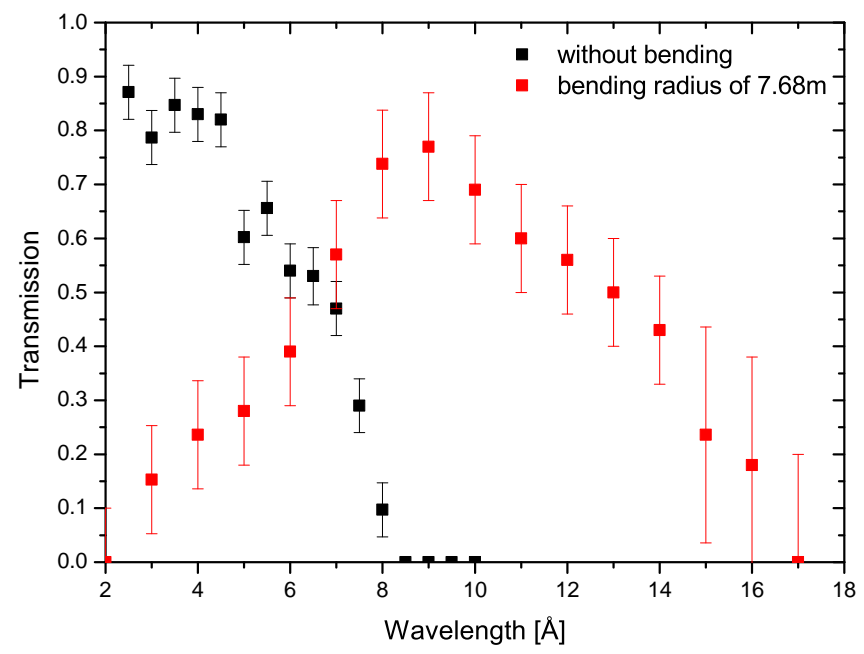

Figure 5: Measured wavelength depending transmission of a collimated white neutron beam through an analyzer without bending and with a bending radius of $7.9 \mathrm{~m}$. 